\title{
Strömgren and $\mathrm{H} \beta$ photometry of $\mathrm{O}$ and $\mathrm{B}$ type stars in star-forming regions
}

\section{Carina Spiral Feature ${ }^{\star, \star \star}$}

\author{
N.T. Kaltcheva ${ }^{1,2}$, E.H. Olsen ${ }^{2}$, and J.V. Clausen ${ }^{2}$ \\ 1 Department of Astronomy, University of Sofia, 5 James Bourchier Avenue, 1164 Sofia, Bulgaria \\ e-mail: nadia@phys.uni-sofia.bg \\ 2 Niels Bohr Institute for Astronomy, Physics and Geophysics, Juliane Maries Vej 30, DK-2100 Copenhagen Ø, Denmark \\ e-mail: eho,jvc@astro.ku.dk
}

Received April 14; accepted July 14, 2000

\begin{abstract}
Strömgren and $\mathrm{H} \beta$ photometry of $\mathrm{O}$ and $\mathrm{B}$ type stars, generally brighter than $9.5 \mathrm{mag}$ is reported for the field of the Carina Spiral Feature. The observations are based on the PPM catalogue identifications and are designed to improve the completeness of the existing uvby $\beta$ data for the bright early-type stars in the field. We present new uvby photometry for 283 stars and $\mathrm{H} \beta$ photometry for 225 of them. These observations are part of an ongoing effort to study the structure of selected star-forming regions in the Milky Way by means of uvby $\beta$ photometry. A comparison of the new data to other uvby $\beta$ data sets for this field is presented.
\end{abstract}

Key words: stars: early type - open clusters and associations: Carina Spiral Feature

\section{Introduction}

The importance of the Carina Spiral Feature in understanding the structure of our own Galaxy has been stressed by Bok (1932) and has since then been emphasized by many others. As pointed out by Graham 30 years ago, the Carina section of the MW is the most promising of all fields for the optical study of a single large scale feature of the Galaxy (Graham 1970). The Carina Feature is spread out over a distance of several

Send offprint requests to: N. Kaltcheva

* Based on data from the Strömgren Automatic Telescope of the Copenhagen University Observatory, La Silla.

** Tables 1 and 2 are only available in electronic form at the CDS via anonymous ftp to 130.79 .128 .5 or via http://cdsweb.u-strasbg.fr/Abstract.html kiloparsecs along the line of sight and may either be part of a major spiral arm, or may represent a strong inter-arm link. A large number of prominent young open clusters and $\mathrm{OB}$ associations are found over a wide range of distances and have also been intensively investigated. The Feature is situated beyond 500 pc, i.e. beyond the limits of the Hipparcos survey (ESA, 1997), and so far photometry remains the most efficient tool to study its characteristics. The observations presented in this paper are aimed at improving the completeness of the Strömgren and $\mathrm{H} \beta$ data of $\mathrm{O}$ and $\mathrm{B}$ type stars, generally brighter than $9.5 \mathrm{mag}$. The field under consideration is within 5 degrees from the Galactic plane, bounded between $l=285^{\circ}$ and $l=295^{\circ}$.

\section{Observations and reductions}

The observations were performed with the Strömgren Automatic Telescope (SAT) of the Copenhagen University Observatory at La Silla. The vast majority of the photometry was obtained during 20 nights in December 1997 January 1998. Several stars, omitted during this run were included in our list of targets during December 1998. The selection of the targets is based on the PPM catalogueSouth (Bastian \& Röser 1993), including all stars of spectral types $\mathrm{O}$ and $\mathrm{B}$ in the field. The uvby $\beta$ compilation of Hauck \& Mermilliod (1998) was used to exclude from the list of targets stars with already existing data. Since it would be of interest to compare the new data to other data sets, some stars in common with previous observations were, however, left in the list. In this way, 283 stars for uvby photometry and 225 for $\mathrm{H} \beta$ photometry were included. 77 stars in the final sample are intrinsically luminous stars (Stephenson \& Sanduleak 1971). 
The selection of standard stars, observing procedures and transformations to the standard systems for the two observing runs are described in detail in Kaltcheva \& Olsen (1999) and Kaltcheva et al. (1999) (Papers I and II). All observations in the present catalogue were made by the SAT in its fully-automatic mode. Details about the spectrometer, the observing procedure, and the fullyautomatic mode are given by Olsen $(1993,1994)$.

A circular diaphragm of $17^{\prime \prime}$ was used. The number of photo-electrons counted was 100000 in the $y$-channel and 70000 in the $\beta_{\text {narrow-channel, except for a few of the }}$ faintest stars. The background was measured at each program star at a fixed offset. Before reductions, several sky measurements from a small area of the sky, and contiguous in time, were combined and then used on all stars in the area. Sky measurements contaminated by faint stars were eliminated in this process.

\section{Results}

In Table 1, the catalogue of Strömgren uvby photometry for 283 program stars is presented. The PPM identifications are given in the first column. Columns 2, 4, 6 and 8 give $V, b-y, m_{1}$ and $c_{1}$. Columns $3,5,7$ and 9 present the internal $\mathrm{rms}$ errors of one observation (weight 1 ). In Cols. 10 and 11 the weight of $V$ and the indices are listed. Column 12 gives the number of nights for each star.

Table 2 gives $\mathrm{H} \beta$ photometry for 225 program stars. The PPM identifications are given in the first column, and the photometry, the internal rms errors of one observation (weight 1), the weight of the $\mathrm{H} \beta$ indices and the number of the nights for each star follow. The cross-identification with the HD and LS numbers is available in the last two columns of the tables.

\section{Comparison of the new data to other data sets}

Good agreement between photometric data coming from different observers is an essential issue when studying the structure and characteristics of star-forming regions, both on the basis of photometric diagrams or via the photometry-derived physical stellar parameters. Observing stars from previous lists helps to get more clear insight on the homogeneity of the existing photometric data sets. This allows one to evaluate the errors in the derived stellar parameters when using photometry from different sources. Furthermore, it will eventually permit to homogenize to some extent the existing $u v b y \beta$ photometric data for the field under investigation. In this paragraph we compare our data to other sources. Tables 3 and 4 present this comparison for the $\mathrm{H} \beta$ photometry and for the Vuvby photometry, respectively. The tables give the mean differences between the individual sources and the new data, together with the corresponding standard deviations (from the mean difference) in $\beta, V, b-y$, $m_{1}$ and $c_{1}$ for each source. The first columns show the number of stars in common involved in the comparison. The sources of the corresponding photometric data sets are listed in the last columns.

The comparison of the new data to the homogenized data from the Hauck \& Mermilliod (1998) compilation in the sense (HM minus this paper) is given in Fig. 1. Figures 2 to 5 present the comparison of the data obtained in this paper to the individual sources used in the HM compilation for the considered field, in the sense (others minus this paper). The agreement between $\mathrm{H} \beta$ photometry from HM and the present paper is in general good (Fig. 1). The mean differences Delta $\beta=\mathrm{H} \beta-\mathrm{H} \beta$ (SAT) listed in Table 3 are below 0.010 mag for the majority of the sources and the comparison to the individual sources present small systematic trends of \pm 0.03 mag only in two cases. For some sources the $V$ magnitudes show a systematic shift in the zero point of as much as $0.03 \mathrm{mag}$ (Fig. 2). This shift is more significant towards the brightest stars $(V<7 \mathrm{mag})$ and does not obviously depend of the colour term $(b-y)$ (Fig. 3$)$. The largest disagreements are with Hill \& Perry (1969) and Eggen (1983): 0.032 and 0.019 mag, respectively. The agreement in $(b-y)$ is within the photometric errors, although for some sources a systematic difference of $0.01 \mathrm{mag}$ may exist (Fig. 4). The $m_{1}$ and $c_{1}$ colour differences obtained in the present paper show a good agreement with Crawford et al. (1971), Schneider \& Weiss (1988) and Kaltcheva \& Georgiev (1994) (Fig. 5). There is a discrepancy of up to -0.02 in $m_{1}$ and up to $0.02 \mathrm{mag}$ in $c_{1}$ with the other sources. Dealing with $\mathrm{O}$ and $\mathrm{B}$ luminous stars, one should keep in mind the variability in light among them (Abt 1957), which also contributes to the scatter between the different sets. Spectral anomalies can also cause large mean errors in magnitude or colour indices (Young 1974; Manfroid 1985; Franco 1994).

The comparisons in Tables 3 and 4 suggest differences in the precision and accuracy of the different data sets. The consistency of different uvby data sets has been already discussed by Manfroid \& Sterken (1987), who stressed the need for a more strictly defined standard system and for closely matching instrumental system. The indication of a small systematic difference in $m_{1}$ and $c_{1}$ is slightly worrying. A similar difference in $c_{1}$ in a large data set has been previously noticed by Franco (1994). Recently, Crawford (1999a) also presented a comparison between uvby data from different publications, showing differences in $m_{1}$ and $c_{1}$ up to $\pm 0.05 \mathrm{mag}$. He also pointed out the reasons that can lead to systematic errors (Crawford 1999b). An additional source may be not applying a correction of -0.008 in $c_{1}$ to a large set of the southern standards (cf. Olsen 1983). In the case of photometry in star-forming regions, the discrepancy may also 

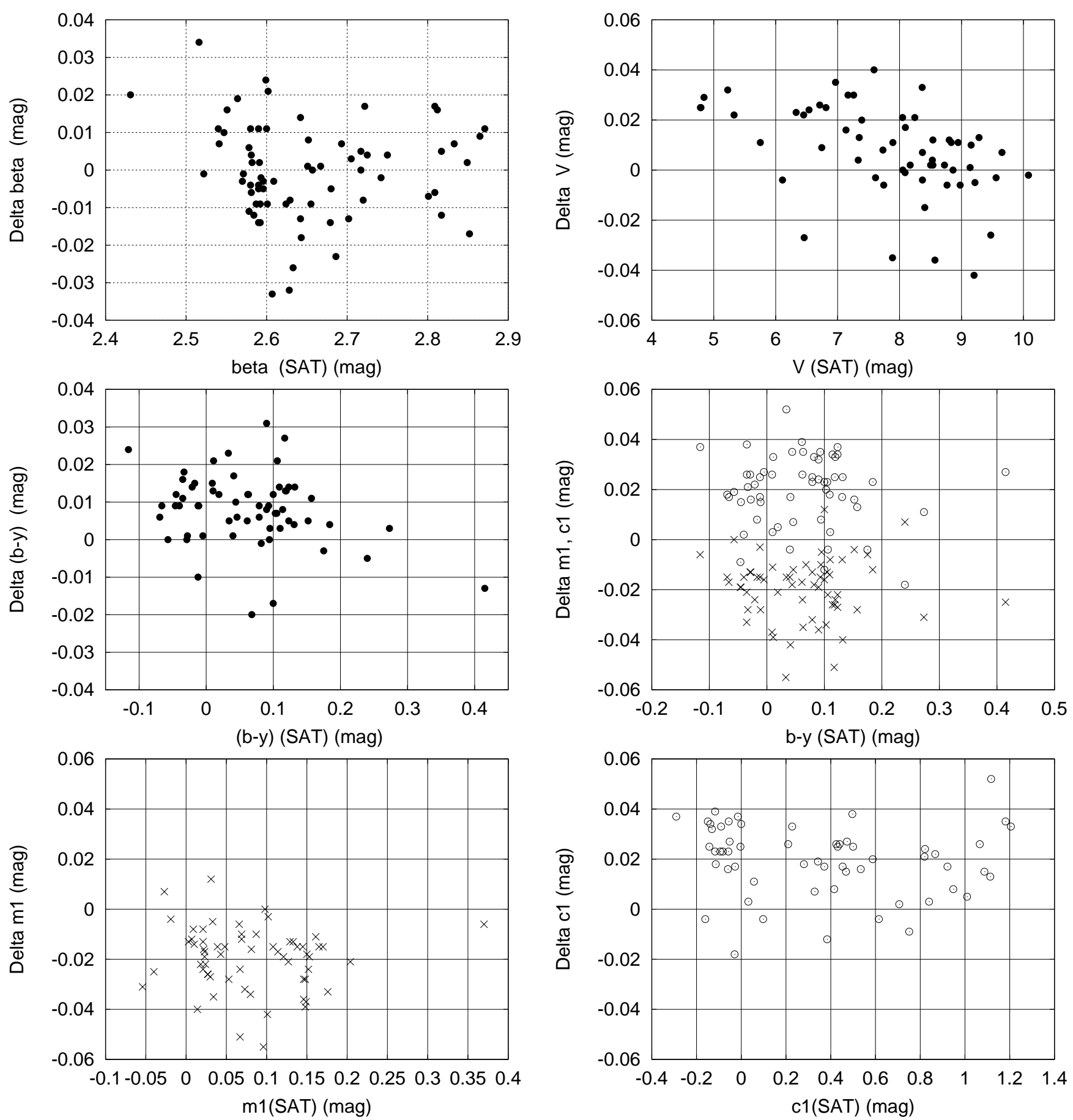

Fig. 1. The differences in the $V$ mag and the photometric indices in the sense (HM-SAT) plotted vs. the SAT values. Crosses are used for $m_{1}$ and open symbols for $c_{1}$

be due to the difficulties in selecting a suitable set of primary and secondary uvby standards among luminous and reddened OB stars (cf. Paper I).

The systematic differences between the existing data sets lead to systematic differences in the photometryderived stellar parameters. The ideal case to compare data only internally is often not possible. In this event the possible inconsistence should be carefully estimated. If the photometric diagrams including $c_{1}$ and $m_{1}$ are used, the differences in the photometry, mentioned above, can be easily misinterpreted in terms of luminosity or metallicity. In case of calculating the reddening, a systematic differences of 0.01 in $b-y$ and 0.02 in $c_{1}$ lead to a difference of 0.01 in $E(b-y)$. In calculating the absolute magnitude $M_{V}$, which is a function of $\beta$ and $c_{0}$, the $\beta$ indices are the most critical parameter. A systematic difference 

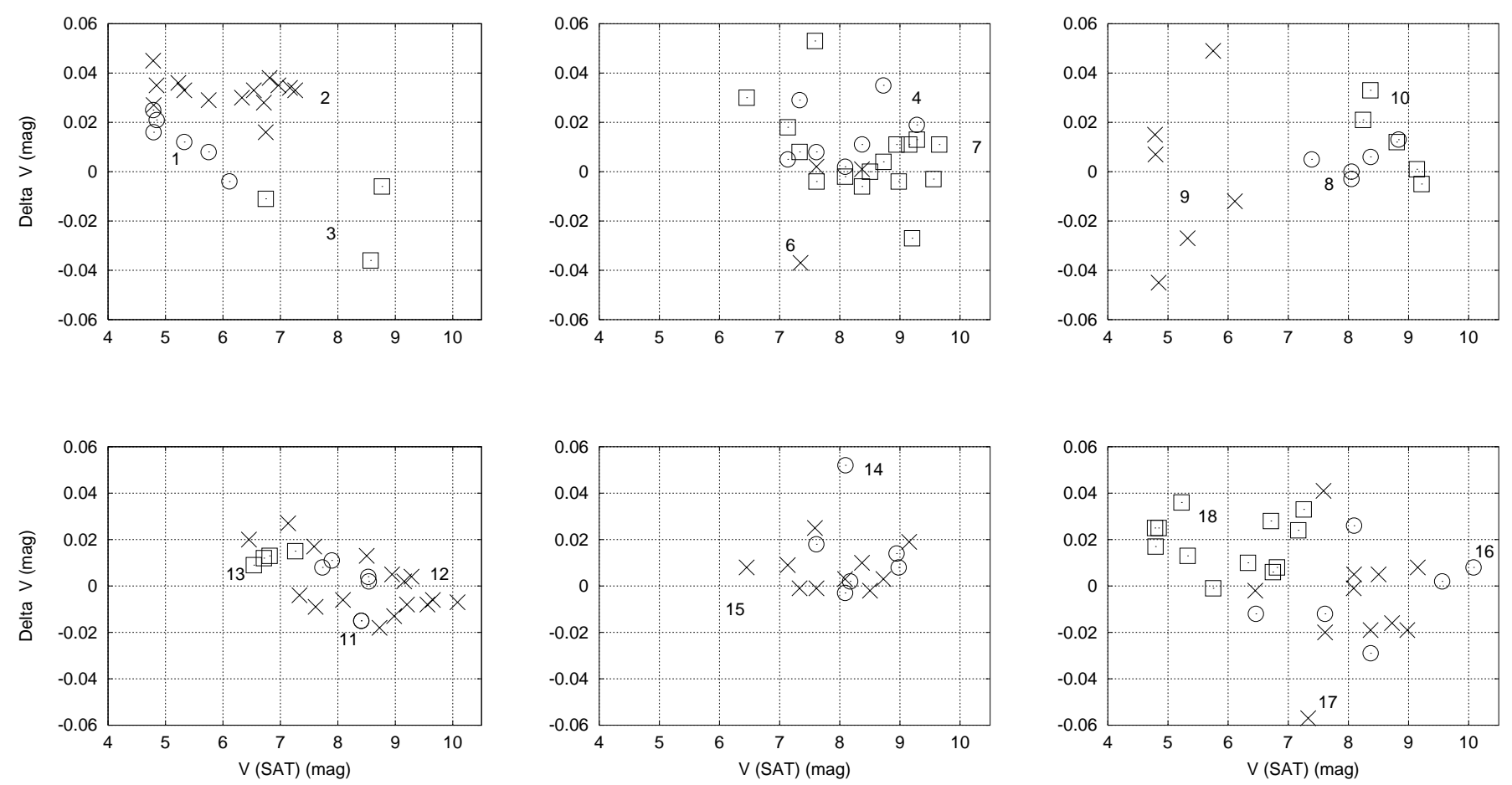

Fig. 2. The differences $V-V(\mathrm{SAT})$ plotted vs. $V(\mathrm{SAT})$ for the sources listed in Table 4
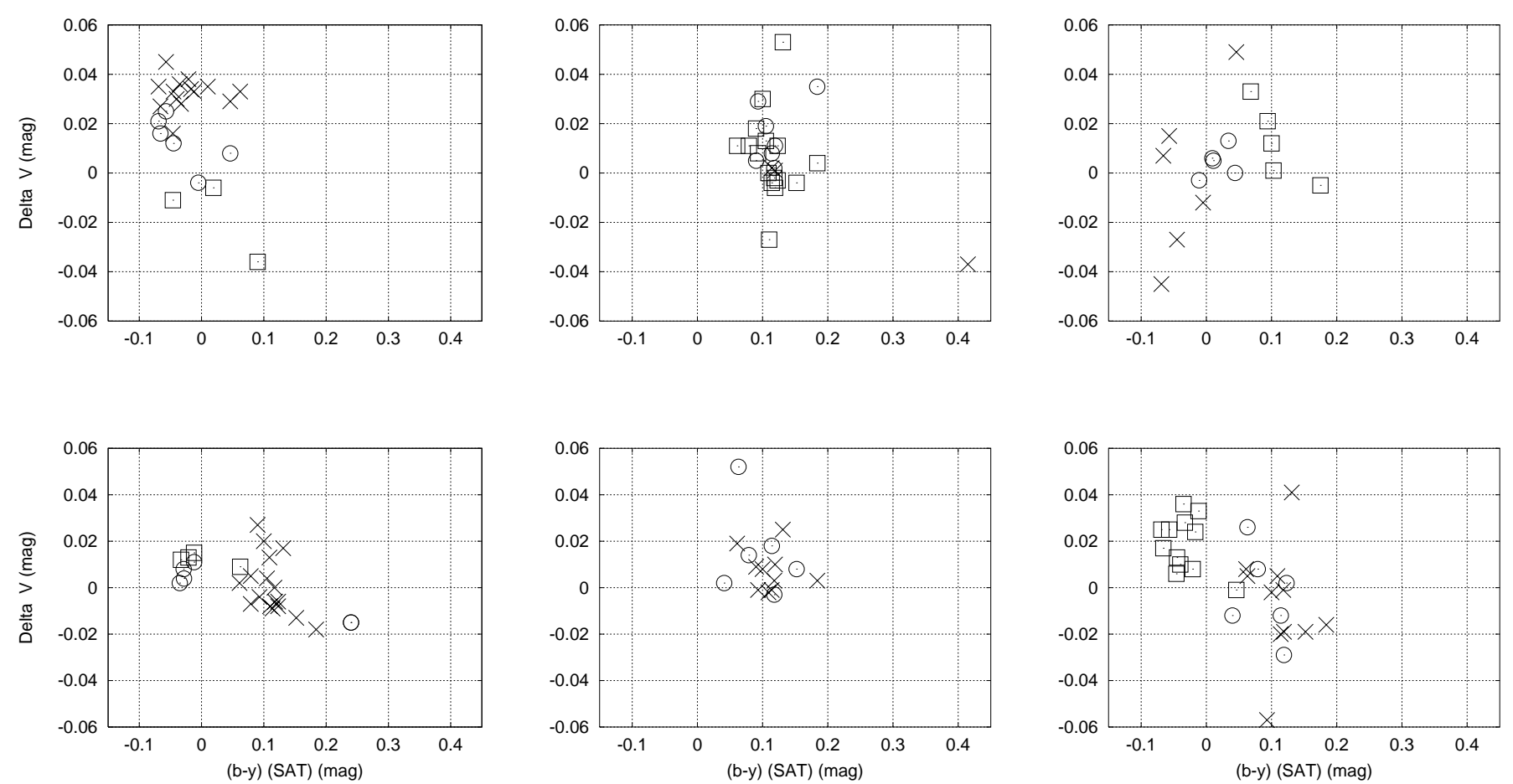

Fig. 3. Difference $V-V(\mathrm{SAT})$ plotted vs. $(b-y)(\mathrm{SAT})$ for the sources listed in Table 4 . The symbols are the same used in Fig. 2 

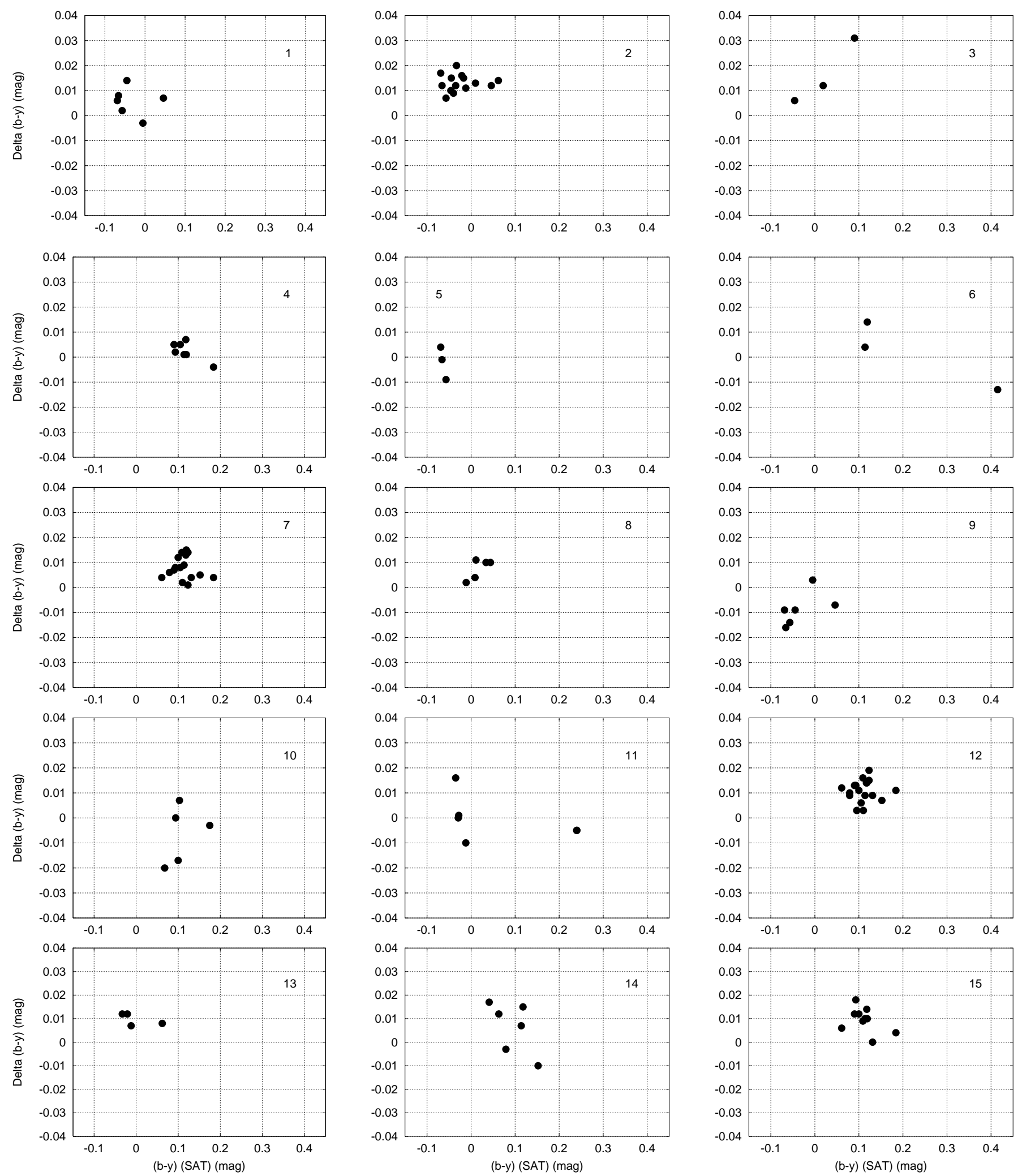

Fig. 4. The differences Delta $(b-y)=(b-y)-(b-y)$ (SAT) plotted vs. $(b-y)$ (SAT) for the sources listed in Table 4 . The individual sources of the photometry are shown at the panels 

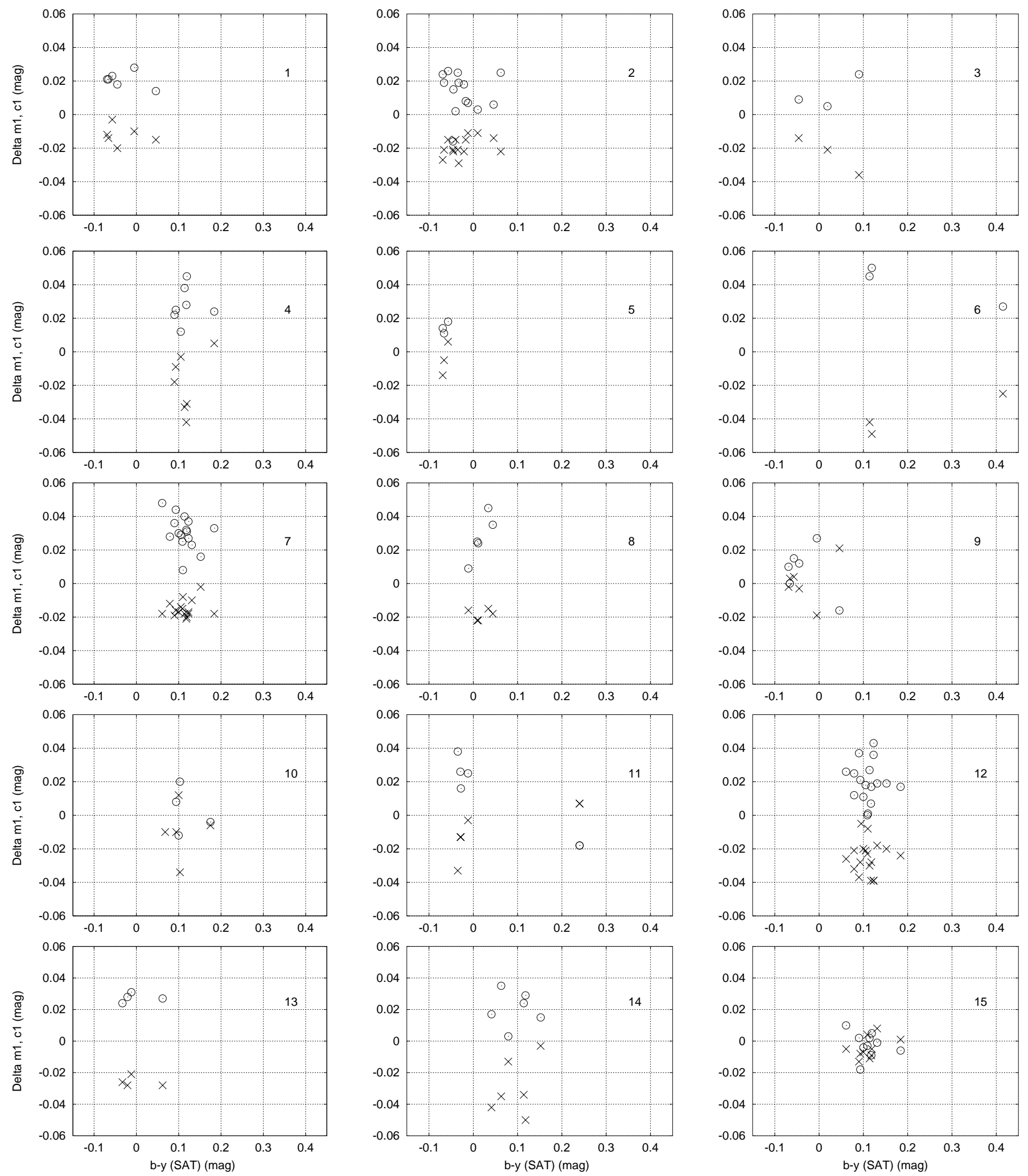

Fig. 5. The differences Delta $m_{1}, c_{1}$ equal to $m_{1}-m_{1}$ (SAT) (crosses) and $c_{1}-c_{1}$ (SAT) (open symbols) plotted vs. $b-y(\mathrm{SAT})$ for the sources listed in Table 4 . The individual sources of the photometry are shown at the panels 
Table 3. Comparison of $\beta$ values obtained in this paper to other authors

\begin{tabular}{rrrl}
\hline$N$ & $D(\beta)$ & $D(\beta)$ & Source \\
$\beta$ & & s.d. & \\
\hline 14 & 0.002 & 0.012 & Hill G., Perry C.L., 1969, AJ 74, 1011 \\
15 & -0.022 & 0.015 & Graham J.A., 1970, AJ 75, 703 \\
6 & -0.005 & 0.017 & Crawford D.L., Barnes J.V., Golson J.C., 1971, AJ 76, 621 \\
3 & -0.003 & 0.009 & Feinstein A., 1974, MNRAS 169, 171 \\
13 & -0.010 & 0.017 & Deutschman W.A., Davis R.J., Schild R.E., 1976, ApJS 30, 97 \\
7 & 0.000 & 0.004 & Gronbech B., Olsen E.H., 1977, A\&AS 27, 443 \\
22 & 0.002 & 0.019 & Klare G., Neckel T., 1977, A\&AS 27, 215 \\
8 & -0.031 & 0.034 & Eggen O.J., 1978, AJ 83, 288 \\
3 & -0.004 & 0.002 & Sinnerstad U., 1980, A\&AS 40, 395 \\
3 & 0.009 & 0.011 & Eggen O.J., 1981, ApJ 246, 817 \\
3 & -0.017 & 0.019 & Lindroos K.P., 1983, A\&AS 51, 161 \\
13 & -0.005 & 0.010 & Eggen O.J., 1983, MNRAS 204, 377 \\
15 & -0.004 & 0.011 & Perry C.L., Landolt A.U., 1986, AJ 92, 844 \\
5 & 0.004 & 0.007 & Landolt A.U., Perry C.L., Levato O.H., Malaroda S.M., 1990, AJ 100, 695 \\
4 & -0.008 & 0.009 & Stetson P.B., 1991, AJ 102, 589 \\
6 & 0.012 & 0.010 & Knude J., 1992, A\&AS 92, 841 \\
10 & -0.007 & 0.010 & Kaltcheva N.T., Georgiev L.N., 1994, MNRAS 269, 289 \\
19 & 0.007 & 0.022 & Corradi W.J.b., Franco G.A.P., 1995, A\&AS 112, 95 \\
\hline
\end{tabular}

Table 4. Comparison of $V, b-y, m_{1}$ and $c_{1}$ values obtained in this paper to other authors

\begin{tabular}{|c|c|c|c|c|c|c|c|c|c|}
\hline $\begin{array}{l}N \\
V u v\end{array}$ & $D(V)$ & $\begin{array}{r}D(V) \\
\text { s.d. }\end{array}$ & $D(b-y)$ & $\begin{array}{r}D(b-y) \\
\text { s.d. }\end{array}$ & $D\left(m_{1}\right)$ & $\begin{array}{r}D\left(m_{1}\right) \\
\text { s.d. }\end{array}$ & $D\left(c_{1}\right)$ & $\begin{array}{r}D\left(c_{1}\right) \\
\text { s.d. }\end{array}$ & Source \\
\hline 6 & 0.013 & 0.010 & 0.006 & 0.006 & -0.012 & 0.006 & 0.021 & 0.005 & 1. Gronbech B., Olsen E.H., 1976, A\&AS 25, 213 \\
\hline 14 & 0.032 & 0.007 & 0.013 & 0.003 & -0.019 & 0.006 & 0.013 & 0.012 & 2. Hill G., Perry C.L., 1969, AJ 74, 1011 \\
\hline 3 & -0.018 & 0.016 & 0.016 & 0.013 & -0.024 & 0.011 & 0.013 & 0.010 & 3. Vogt N., Faundez M.A., 1979, A\&AS 36, 477 \\
\hline 7 & 0.016 & 0.012 & 0.002 & 0.004 & -0.019 & 0.017 & 0.028 & 0.011 & 4. Eggen O.J., 1978, AJ 83, 288 \\
\hline 3 & & & -0.002 & 0.007 & -0.004 & 0.010 & 0.014 & 0.003 & 5. Sinnerstad U., 1980, A\&AS 40, 395 \\
\hline 3 & -0.011 & 0.022 & 0.002 & 0.014 & -0.039 & 0.012 & 0.041 & 0.012 & 6. Lindroos K.P., 1983, A\&AS 51, 161 \\
\hline 16 & 0.007 & 0.018 & 0.009 & 0.004 & -0.015 & 0.005 & 0.030 & 0.010 & 7. Perry C.L., Landolt A.U., 1986, AJ 92, 844 \\
\hline 5 & 0.004 & 0.006 & 0.007 & 0.004 & -0.019 & 0.003 & 0.028 & 0.013 & 8. Schneider H., 1987, A\&AS 71, 147 \\
\hline 6 & -0.002 & 0.033 & -0.009 & 0.007 & 0.001 & 0.013 & 0.008 & 0.015 & $\begin{array}{l}\text { 9. Crawford D.L., Barnes J.V., Golson J.C., 1971, } \\
\text { AJ 76, } 621\end{array}$ \\
\hline 5 & 0.012 & 0.015 & -0.007 & 0.011 & -0.010 & 0.016 & 0.003 & 0.014 & 10. Schneider H., Weiss W.W., 1988, A\&AS 75, 353 \\
\hline 5 & 0.002 & 0.010 & 0.000 & 0.009 & -0.011 & 0.014 & 0.017 & 0.021 & $\begin{array}{l}\text { 11. Landolt A.U., Perry C.L., Levato O.H., Malaroda S.M., } \\
\text { 1990, AJ 100, } 695\end{array}$ \\
\hline 18 & 0.000 & 0.012 & 0.011 & 0.004 & -0.025 & 0.010 & 0.020 & 0.019 & 12. Corradi W.J.b., Franco G.A.P., 1995, A\&AS 112, 95 \\
\hline 4 & 0.012 & 0.002 & 0.010 & 0.003 & -0.026 & 0.003 & 0.028 & 0.003 & 13. Stetson P.B., 1991, AJ 102, 589 \\
\hline 6 & 0.015 & 0.020 & 0.006 & 0.011 & -0.030 & 0.018 & 0.020 & 0.011 & 14. Knude J., 1992, A\&AS 92, 841 \\
\hline 10 & 0.007 & 0.009 & 0.009 & 0.005 & -0.004 & 0.007 & -0.002 & 0.008 & 15. Kaltcheva N.T., Georgiev L.N., 1994, MNRAS 269, 289 \\
\hline 6 & -0.003 & 0.019 & & & & - & & & $\begin{array}{l}\text { 16. Deutschman W.A., Davis R.J., Schild R.E., 1976, } \\
\text { ApJS 30, } 97\end{array}$ \\
\hline 12 & -0.007 & 0.024 & - & - & - & - & - & & 17. Klare G., Neckel T., 1977, A\&AS 27, 215 \\
\hline 12 & 0.019 & 0.012 & - & - & - & - & - & & 18. Eggen O.J., 1983, MNRAS 204, 377 \\
\hline
\end{tabular}

of $0.03 \mathrm{mag}$ in $\beta$, leads to a systematic differences in the corresponding $M_{V}$ values of $0.3 \mathrm{mag}$. A systematic difference from the standard system of $+0.02 \pm 0.02 \mathrm{mag}$ in $c_{1}$ alone leads to overestimation of $M_{V}$ of $0.15 \mathrm{mag}$ and to overestimation of the distance of about $5 \%$.

The structure of the Carina Spiral Feature field based on photometry presented here will be discussed in a forthcoming paper.

Acknowledgements. This research has been supported by the Danish Natural Science Research Council. This research has made use of the Simbad database, operated at CDS, Strasbourg, France.

\section{References}

Abt H., 1957, ApJ 126, 138

Bastian U., Röser S., 1993, Catalogue of Positions and Proper Motions - South, Astronomisches Rechen-Institut, Heidelberg

Bok B.J., 1932, Harvard Repr., No. 77

Crawford D.L., 1999a, in Craine D., Tucker R., Barnes J. (eds.), ASP Conf. Ser. 189, Astron. Soc. Pac., San Francisco, p. 6

Crawford D.L., 1999b, in Craine, D., Tucker R., Barnes J. (eds), ASP Conf. Ser. 189, Astron. Soc. Pac., San Francisco, p. 3

Crawford D.L., Barnes J.V., Golson J.C., 1971, AJ 76, 621

Graham J.A., 1970, IAU Symp. 38, 262

Eggen O.J., 1983, MNRAS 204, 377

ESA 1997, The Hipparcos Catalogue, ESA SP-1200 
Franco G.A.P., 1994, A\&AS 104, 9

Hauck B., Mermilliod M., 1998, A\&AS 129, 143

Hill G., Perry C.L., 1969, AJ 74, 1011

Kaltcheva N.T., Georgiev L.N., 1994, MNRAS 269, 289

Kaltcheva N.T., Olsen E.H., 1999, A\&A 352, 600 (Paper I)

Kaltcheva N.T., Olsen E.H., Clausen J.V., 1999, A\&A 352, 605 (Paper II)

Manfroid J., 1985, Traitement Numérique des Données Photométriques, Université de Liège
Manfroid J., Sterken C., 1987, A\&AS 71, 539

Olsen E.H., 1983, A\&AS 54, 55

Olsen E.H., 1993, A\&AS 102, 89

Olsen E.H., 1994, A\&AS 106, 257

Schneider H., Weiss W.W., 1988, A\&AS 75, 353

Stephenson C.B., Sanduleak N., 1971, Luminous Stars in the Southern Milky Way, Publ. Warner Swasey Obs. 1, 1

Young A.T., 1974, in Methods of Experimental Physics 12A, Carleton N. (ed.). Academic Press, NY, p. 123 